\title{
Circadian rhythms and cognition in schizophrenia ${ }^{\dagger}$
}

\author{
Katharina Wulff and Eileen Joyce
}

\section{Summary}

Cognitive impairment is a core feature of schizophrenia. Evidence in this Journal suggests that disrupted circadian synchronisation of sleep-wake phases may be one cause. Circadian synchronisation depends on the external input of light-dark cues and the integrity of central nervous system circadian oscillators; these may represent new interventional targets to improve cognition.

\section{Declaration of interest}

None.
Katharina Wulff (pictured) is a senior research scientist at the University of Oxford Nuffield Laboratory of Ophthalmology, with expertise in sleep and circadian neuroscience. Eileen Joyce is Professor of Neuropsychiatry at University College London Institute of Neurology and consultant neuropsychiatrist at the National Hospital for Neurology and Neurosurgery.

Cognitive impairment is a sine qua non of schizophrenia - even patients with apparently intact neuropsychological function are likely to have declined from a higher premorbid level. ${ }^{1}$ The evidence indicates that this is generalised in nature ${ }^{2}$ and can affect many abilities important for effective negotiation of the world, including learning, remembering, planning and strategy formation. These higher-order cognitive functions are underpinned by more specific processes such as working memory, inhibition, memory consolidation and information-processing speed, all of which are impaired in schizophrenia. Cognitive impairment is present at psychosis onset and is a strong predictor of later social and occupational dysfunction. ${ }^{3}$ Thus there is a recognised and urgent need for interventions targeting this disability and, with this in mind, all potentially treatable sources of cognitive impairment deserve examination.

\section{Internal representation of circadian time}

It is well established that cognitive ability is dependent on the time of day, being adapted to the day-night cycle and thereby linked to phases of sleep and wakefulness. However, this periodicity does not automatically follow environmental or sleep-wake changes. Instead, it is driven by an endogenous circadian clock that oscillates with a period of approximately $24 \mathrm{~h}$ (about $1=$ circa diem) and which is still evident when individuals are isolated from external periodic signals, or 'zeitgebers', such as light, mealtimes or social activities associated with the time of day. Under such 'freerunning' conditions, all circadian rhythms continue to oscillate, albeit with a tendency to drift away from the 24-hour period. The reintroduction of external circadian signals resynchronises the pacemaker's clockwork and the rhythms that it drives to the 24-hour day, ensuring that the body is prepared to anticipate predictable challenges intrinsically tied to day and night. To achieve optimal temporal alignment between the endogenous clock and the outside world, organisms evolved intracellular

†'see pp. 269-276, this issue. molecular-genetic mechanisms that drive the oscillatory clock machinery, ${ }^{4}$ with the aid of delayed negative feedback loops based on rhythmic transcription, translation and post-translational processes. This oscillatory clock machinery drives timed expression of many downstream target genes and proteins, and only then do their products lead to the rhythmic output of cells such as neuronal firing important for neurotransmitter release or enzymatic metabolic cycles.

A relatively new finding is that the circadian master clock, the hypothalamic suprachiasmatic nuclei (SCN), is not the only oscillator in the brain. Local semi-autonomous oscillators, capable of generating self-sustained circadian oscillations in individual cells independently of the SCN, exist in a number of brain tissues, including the hippocampus, cerebral cortex and cerebellum. ${ }^{5,6}$ Although these oscillators employ the same intracellular clock components as the SCN, they still require input from the SCN to maintain synchrony among individual cells; hence they are not master clocks. As a consequence of this extra layer of complexity, phase coherence among individual brain oscillators and between oscillators and sleep/wake episodes is required. ${ }^{4}$ The key role of the SCN in this context is to is to synchronise 24-hour rhythms in periodic physiological processes, including sleep-wake phases, via its direct innervations from the retina, and provide accurate time cues to local clockworks, thereby imposing internal phase coherence on oscillators in the brain and periphery. The role of the subsidiary oscillators in local brain areas is to time rhythmic expression of genes and proteins appropriately in anticipation of the different synaptic signalling demands during wakefulness (e.g. acquisition and processing of external stimuli) and sleep (e.g. synaptic plasticity of hippocampal neurons). Considering the control that circadian oscillators exert over neural function, temporary or chronic disruption at any level of the clockwork by genetic, pharmacological and environmental factors such as jet lag, shift work and illness, will lead to a loss of phase coherence among local oscillators and between oscillators and sleep-wake phases, thereby causing poor sleep quality and suboptimal cognitive performance. ${ }^{7}$

\section{Disrupted circadian rhythmicity and compromised cognitive performance in schizophrenia}

The study by Bromundt and colleagues ${ }^{8}$ in this month's Journal provides important new information concerning the link between impairments in neuropsychological function and disrupted circadian rhythms in schizophrenia and, in doing so, points to 
new strategies for cognitive remediation of this disorder. The authors assessed the 24-hour activity of well-characterised patients with schizophrenia by asking them to wear 'actigraphs', which resemble wrist watches, over a 3-week period. They also measured melatonin secretion before and after sleep on several occasions during this time to provide a biological marker of circadian master clock activity. Although the patient group was relatively small $(n=14)$, the effort involved in producing such valuable longitudinal data should not be underestimated and, further, the results are clear. The 24-hour activity of all patients was appropriately entrained to light-dark phases. Thus these patients slept at night and were awake during the day and therefore did not exhibit the gross disruptions of circadian activity sometimes seen in people with mental health problems whereby normal day-night activity is persistently irregular. Instead, circadian activity was disrupted in a more subtle way. Half of the patients had increased night-time activity, shorter and more fragmented time spent asleep and more daytime sleepiness. In addition, whereas the patients with normal day-night activity showed synchronised melatonin rhythms, in the others melatonin release was out of phase, beginning after their usual bedtime (rather than before) and extending beyond their waking time.

Cognition was measured before and after the actigraphy study and was essentially stable. The group with normal circadian rhythms performed within the normal range according to published population data for the tests employed. These focused on executive functions, examined by the ability to call up from memory semantically related words (verbal fluency), inhibit a pre-potent response (the Stroop Test) and rapidly switch attention from one category to another (the Trail Making Test). Compared with the normal group, patients with disrupted circadian rhythms were worse on all but one of these cognitive functions.

One explanation of these finding is that the impaired group was simply more ill. Importantly, however, across all patients there were large associations between the ratio of day-night activity and the cognitive measures, with effect sizes ranging from 0.66 to 0.83 , but no such associations with symptom severity or length of illness. This suggests that there is a specific relationship between abnormalities of circadian rhythm and impaired cognition in schizophrenia and that this is significant for a subgroup of patients.

\section{What is the origin of disrupted circadian rhythms in schizophrenia?}

All patients were receiving antipsychotic medication and some adjunctive medications, but this did not seem to explain the group differences; nor did illness duration or other variables such as age and body mass index. One possibility is that the patients with abnormal sleep-wake activity were not being exposed to strong enough zeitgebers because of social withdrawal or lack of daytime structure. There is a hint from the study that this might apply as more of the patients with disrupted circadian rhythms were outpatients and therefore less likely to have a daily routine of the kind imposed on in-patients. Another explanation is neurobiological; that is, people with schizophrenia may have intrinsically unstable circadian oscillators. For example, there may be impaired circadian synchronisation between cellular networks of local brain areas participating in sleep-wake regulation and cognition which might arise from natural genetic variation, as seen in animal models of neuronal PAS-domain protein-2 (NPAS2) deficiency. ${ }^{9}$ Abnormalities of local oscillators residing in the hippocampus and frontal cortex are candidate mechanisms as both are structurally abnormal in schizophrenia and highly implicated in mediating the cognitive impairment associated with the disorder. In addition, there is some, albeit modest, evidence for an association between schizophrenia and a common polymorphism of the NPAS2 gene. ${ }^{10}$ Conflicting zeitgeber signals (e.g. light exposure at night) would further hamper abnormal neurobiological temporal adaptation processes, while daily clinical routines would strengthen temporal synchronisation. Thus, whether the primary mechanism is environmental or neurobiological, patients with schizophrenia with disrupted circadian activity are at a disadvantage because of its effect on already compromised cognitive function, which may lead to a vicious cycle of failure in the performance of everyday functions, further social withdrawal and reduced zeitgeber exposure. Recognising sleep-wake disturbances and establishing their underlying causes is therefore of great importance.

\section{How can an understanding of sleep-wake disturbances in schizophrenia lead to therapies that might improve cognition?}

There is already evidence ${ }^{11}$ to suggest that exogenous melatonin can help with the type of sleep-wake abnormalities described in the study by Bromundt and colleagues. Procedures comparing melatonin onset (measured in saliva or urine) and sleep-wake timing (measured with actigraphy) are straightforward to conduct and could provide useful information at an individual level by assessing circadian phase relationships and separating out internal desynchronisation and misalignment between internal phase and environmental light-dark phase. The results could then be used to inform rehabilitation strategies and, if indicated, introduce personalised chronotherapy, which might include individually timed melatonin administration and/or exposure to bright light. ${ }^{11}$ The ability to regulate circadian activity to encourage consolidated sleep patterns may thus prove a valuable intervention that targets one source of cognitive difficulties in patients with schizophrenia.

Katharina Wulff, PhD, Sleep and Circadian Neuroscience Group, Nuffield Laboratory of Ophthalmology, University of Oxford. Eileen Joyce, MA, PhD, MRCP, FRCPsych, Institute of Neurology, University College London, UK

Correspondence: Eileen Joyce, Professor of Neuropsychiatry, Box 19, The National Hospital for Neurology and Neurosurgery, Queen Square, London WC1N 3BG, UK. Email: e.joyce@ion.ucl.ac.uk

First received 19 Oct 2010, final revision 11 Nov 2010, accepted 2 Feb 2011

\section{Funding}

K.W. is supported by the Oxford Biomedical Research Centre funded by the National Institute of Health Research (NIHR). E.J. is supported by the Raymond Way Fund. K.W. and E.J. were funded by a Wellcome Trust project grant (No. 076067) for work leading to this editorial.

\section{References}

1 Wilk CM, Gold JM, McMahon RP, Humber K, Iannone VN, Buchanan RW. No, it is not possible to be schizophrenic yet neuropsychologically normal. Neuropsychology 2005; 19: 778-86.

2 Dickinson D, Ragland JD, Gold JM, Gur RC. General and specific cognitive deficits in schizophrenia: Goliath defeats David? Biol Psychiatry 2008; 64 823-7. 
3 Leeson VC, Barnes TR, Hutton SB, Ron MA, Joyce EM. IQ as a predictor of functional outcome in schizophrenia: a longitudinal, four-year study of first-episode psychosis. Schizophr Res 2009; 107: 55-60.

4 Takahashi JS, Hong HK, Ko CH, McDearmon EL. The genetics of mammalian circadian order and disorder: implications for physiology and disease. Nat Rev Genet 2008; 9: 764-75.

5 Maywood ES, Fraenkel E, McAllister CJ, Wood N, Reddy AB, Hastings MH, et al. Disruption of peripheral circadian timekeeping in a mouse model of Huntington's disease and its restoration by temporally scheduled feeding. J Neurosci 2010; 30: 10199-204.

6 Wang LM, Dragich JM, Kudo T, Odom IH, Welsh DK, O'Dell TJ, et al. Expression of the circadian clock gene Period2 in the hippocampus: possible implications for synaptic plasticity and learned behaviour. ASN Neuro 2009; 1: e00012.
7 Kyriacou CP, Hastings MH. Circadian clocks: genes, sleep, and cognition. Trends Cogn Sci 2010: 14: 259-67.

8 Bromundt V, Köster M, Georgiev-Kill A, Opwis K, Wirz-Justice A, Stoppe G, et al. Sleep-wake cycles and cognitive functioning in schizophrenia. Br J Psychiatry 2011; 198: 269-276.

9 Dudley CA, Erbel-Sieler C, Estill SJ, Reick M, Franken P, Pitts S, et al. Altered patterns of sleep and behavioral adaptability in NPAS2-deficient mice. Science 2003; 301: 379-83.

10 Mansour HA, Talkowski ME, Wood J, Chowdari KV, McClain L, Prasad K, et al. Association study of 21 circadian genes with bipolar I disorder, schizoaffective disorder, and schizophrenia. Bipolar Disord 2009; 11 701-10.

11 Bjorvatn B, Pallesen S. A practical approach to circadian rhythm sleep disorders. Sleep Med Rev 2009; 13: 47-60.

\section{psychiatry in pictures}

\section{Before the Meeting}

\section{Sarvenaz Keyhani}

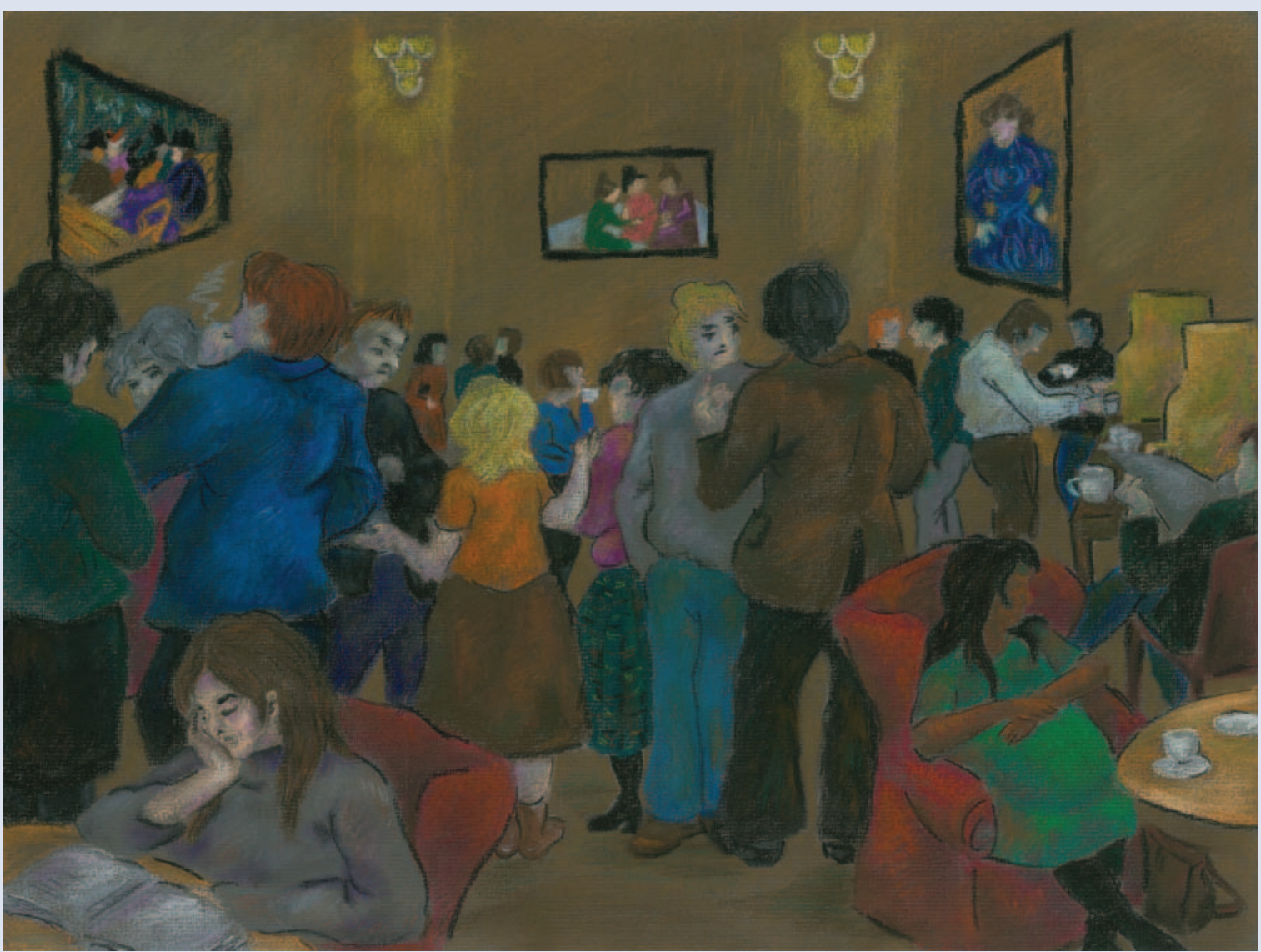

Sarvenaz Keyhani is a staff grade psychiatrist working in general adult psychiatry in London. She has been painting from a young age and had an exhibition in Tehran in 2000. She continues to paint alongside practising psychiatry and believes there is a strong relationship between painting and psychiatry, art therapy being just one example.

The painting Before the Meeting was done in 2008, after the artist attended a psychiatry meeting in Wessex, where she was having her psychiatry training. It is chalk pastel on coloured paper of a group of people gathering in a waiting area. It is a realistic painting; the large areas of warm colour, especially the brown, intensify the warm atmosphere of the place, as well as the aroma of the coffee the guests are drinking. The three paintings on the walls are copies of Lautrec's, added here to increase the effect of colour in the painting as well as being a homage to the great painter for his intense realistic vision and impressive use of colour. 